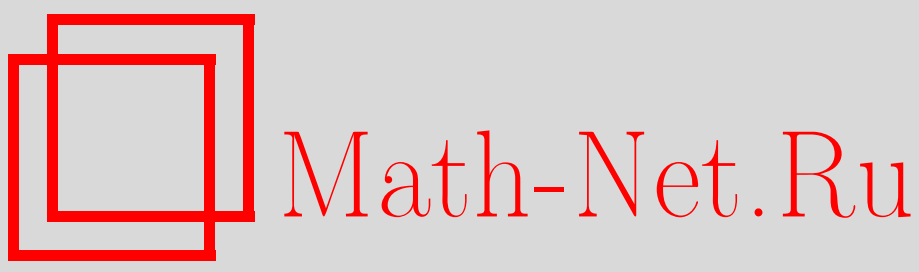

В. А. Мирзоян, Подмногообразия с полупараллельными тензорными полями как огибающие, Матем. сб., 2002, том 193, номер 10, 99-112

DOI: https://doi.org/10.4213/sm686

Использование Общероссийского математического портала Math-Net.Ru подразумевает, что вы прочитали и согласны с пользовательским соглашением

http://www . mathnet.ru/rus/agreement

Параметры загрузки:

IP: 34.229 .45 .116

26 апреля 2023 г., $12: 44: 33$ 


\author{
В. А. Мирзоян
}

\title{
Подмногообразия с полупараллельными тензорными полями как огибающие
}

\begin{abstract}
В пространствах постоянной кривизны в терминах различных классов огибающих дается полное решение проблемы взаимосвязи между подмногообразиями с полупараллельньми тензорными полями, определенньми второй фундаментальной формой с использованием произволњных тензорных операций, и подмногообразиями с соответствующими параллельньми тензорньти полями.

Библиография: 26 названий.
\end{abstract}

\section{§1. Постановка проблемы и ее решение в частных случаях}

На протяжении последних 35-ти лет различные диффференциально-геометрические обобщения симметрических пространств привели к изучению римановых пространств и подмногообразий с параллельньми и полупараллельными структурами [1]-[19]. В качестве таких структур выступают различные тензорные поля такие, как тензор кривизны, тензор Риччи, фундаментальные формы (ф.ф.) $\alpha_{s}(s \geqslant 2)$ подмногообразия и др.

В этом направлении долгое время оставалась нерешенной следуюшая общая проблема: какова взаимосвязь между подмногообразиями с параллельньми тензорными полями и подмногообразиями с полупараллельными тензорньми полями?

В 1990 г. для подмногообразий с полупараллельной второй ф.ф. $\alpha_{2}$ в пространствах постоянной кривизны эта проблема была решена Ю. Г. Лумисте [6]. Для подмногообразий с полупараллельной ф.ф. $\alpha_{s}(s \geqslant 3)$, а также для подмногообразий с полупараллельным тензорным полем, определенным формой $\alpha_{2}$ с использованием только тензорных операций алгебраического характера (тензор кривизны, тензор Риччи, тензор кривизны нормальной связности и вектор средней кривизны подмногообразия являются примерами такого тензорного поля), эта проблема была решена автором в [14]-[20].

В настоящей работе в объемлюшем пространстве постоянной кривизны указанная выше проблема решается в обшем виде для подмногообразия с полупараллельньпи ковариантными производными произвольного тензорного поля, определяемого формой $\alpha_{2}$ с использованием тензорных операций как алгебраического, так и дифференциального характера.

Учитывая итоговьй характер работы, напомним основные определения и приведем ряд известных теорем из работ [6], [14], [18], дающих решение проблемы в частных случаях.

Пусть $M$ является подмногообразием $n$-мерного пространства постоянной кривизны $M_{n}(c)$, и пусть $\nabla$ и $\nabla^{\perp}$ обозначают риманову и нормальную связности на $M$, 
а $\bar{\nabla}=\nabla \oplus \nabla^{\perp}-$ связность Ван дер Вардена-Бортолотти. Для произвольных касательных к $M$ векторных полей $X, Y$ оператор кривизны $\bar{R}(X, Y)$ связности $\bar{\nabla}$ определяется формулой $\bar{R}(X, Y)=\left[\bar{\nabla}_{X}, \bar{\nabla}_{Y}\right]-\bar{\nabla}_{[X, Y]}$. Известно, что эти операторы действуют как дифференцирования тензорной алгебры на $M$.

Тензорное поле $K$ на подмногообразии $M$ называется параллельным, если $\bar{\nabla} K=0$ или, более подробно, если $\bar{\nabla}_{X} K=0$ для любого касательного к $M$ векторного поля $X$. Тензорное поле $K$ называется полупараллельным, если $\bar{R}(X, Y) \cdot K=0$ для любых касательных к $M$ векторных полей $X, Y$.

В $M_{n}(c)$ подмногообразие $M$ называется полупараллельным [1]-[5] (или полусимметрическим [6]-[9]), если его вторая ф.ф. $\alpha_{2}$ полупараллельна. На протяжении последних 16-ти лет этот класс подмногообразий интенсивно исследуется многими авторами (см. работы [4], [5], [7], [8], [10] и цитированную в них литературу). Большой интерес обусловлен тем, что в силу импликации

$$
\bar{R}(X, Y) \cdot \alpha_{2}=0 \Rightarrow R(X, Y) \cdot R=0
$$

( $R$ - тензор кривизны) полупараллельные подмногообразия имеют внутреннюю геометрию полусимметрического риманова пространства, характеризуемого последним условием. Важнейшее свойство полупараллельных подмногообразий описывает

Теорема ЛУмИСТЕ [6]. $B M_{n}(c)$ m-мерное подмногообразие $M$ полупараллельно тогда и только тогда, когда оно является огибающим второго порядка семейства т-мерных параллельных подмногообразий.

Параллельные (или симметрические, что то же самое) подмногообразия характеризуются условием $\bar{\nabla} \alpha_{2}=0$ и, в основном, исчерпьваются стандартньми погружениями симметрических $R$-пространств [11]-[13].

В цикле работ автора [14]-[20] теорема Лумисте обобщалась как в направлении усиления закона огибания, так и в направлении его ослабления. Приведенные ниже определения и сфформулированные теоремы показывают, в каких направлениях происходило это обобшение.

Пусть $M$ и $\widetilde{M}$ - два $m$-мерных подмногообразия в $M_{n}(c)$, имеющих общую точку $x$. Говорят, что $x$ является точкой касания 2-го порядка для $M$ и $\widetilde{M}$, если для каждой кривой $\gamma \in M$, проходящей через $x$, существует кривая $\widetilde{\gamma} \in \widetilde{M}$, также проходящая через $x$ и имеющая с $\gamma$ общую касательную и вектор кривизны. Ю.Г. Лумисте [6] доказал, что касание 2-го поря дка в точке $x$ равносильно совпадению касательных пространств $T_{x}(M)$ и $T_{x}(\widetilde{M})$ и вторых ф.фф. $\alpha_{2 x}$ и $\widetilde{\alpha}_{2 x}$ в этой точке. В последней форме понятие касания 2-го порядка допускает следуюшее обобщение.

ОПРЕДЕЛЕНИЕ 1 . В $M_{n}(c)$ обшая точка $x$ двух $m$-мерных подмногообразий $M$ и $\widetilde{M}$ называется точкой касания $s-2 o(s \geqslant 2)$ порядка, если в этой точке совпадают

1) касательные пространства $T_{x}(M)$ и $T_{x}(\widetilde{M})$,

2) ф.ф. $\alpha_{r}$ и $\widetilde{\alpha}_{r}, r=2, \ldots, s$, подмногообразий $M$ и $\widetilde{M}$ соответственно, т.е. $\alpha_{r x}=\widetilde{\alpha}_{r x}$ или, более подробно, $\alpha_{r x}\left(X_{1}, \ldots, X_{r}\right)=\widetilde{\alpha}_{r x}\left(X_{1}, \ldots, X_{r}\right)$ для любых векторов $X_{1}, \ldots, X_{r} \in T_{x}(M)$. 
Если условие 2) выполняется для любого $s \geqslant 2$, то $x$ называется точкой полного касания подмногообразий $M$ и $\widetilde{M}$.

Фундаментальные формы $\alpha_{r}$ определяются (подробности см. в [8], [14], [16]) по рекуррентной формуле $\alpha_{r}=\bar{\nabla} \alpha_{r-1}(r \geqslant 3)$.

Такое понимание касания $s$-го порядка подмногообразий не совпадает с общепринятым (см. [21; гл. $6, \S 4])$ и выгодно отличается тем, что определяется с использованием только фундаментальных объектов подмногообразий, характеризующих всю их геометрию.

Отметим следующее важное геометрическое следствие: если $x \in M \cap \widetilde{M}$ является точкой касания $(r+1)$-го порядка, то в этой точке совпадают $k$-е $(k=1, \ldots, r)$ соприкасаюшиеся пространства подмногообразий $M$ и $\widetilde{M}$. Действительно, соприкасающиеся пространства определяются следующим образом. Пусть $\left(\left(\alpha_{k}\right)\right)_{x}$ обозначает линейную оболочку нормальных векторов вида $\alpha_{k}\left(X_{1}, \ldots, X_{k}\right)$, где $X_{1}, \ldots, X_{k} \in T_{x}(M)$. Подпространство $N_{1}(x)=\left(\left(\alpha_{2}\right)\right)_{x}$ нормального пространства $T_{x}^{\perp}(M)$ называется первым нормальным пространством подмногообразия $M$ в точке $x$. Нормальные пространства $N_{k}(x)(k \geqslant 2)$ подмногообразия $M$ в точке $x$ определяются по формуле

$$
N_{k}(x)=\left(\left(\alpha_{k+1}\right)\right)_{x} \cap\left(N_{1}(x)+\cdots+N_{k-1}(x)\right)^{\perp},
$$

где $(\cdot)^{\perp}$ обозначает ортогональное дополнение в $T_{x}^{\perp}(M)$. Прямая сумма $T_{x}(M)+$ $N_{1}(x)+\cdots+N_{k}(x)$ называется $k$-м соприкасающимся пространством подмногообразия $M$ в точке $x$. Теперь очевидно, что высказанное выше утверждение справедливо.

ОПРЕДЕЛЕНИЕ $2 . \mathrm{B} M_{n}(c) m$-мерное подмногообразие $M$ назьвается огибающим $s$-го порядка $(s \geqslant 2)$ некоторого семейства $m$-мерных подмногообразий $\{G\}$, если в каждой своей точке оно имеет касание $s$-го порядка с каким-либо подмногообразием этого семейства, причем касание вдоль $M$ переходит от одного подмногообразия семейства к другому. Если $M$ в каждой своей точке имеет полное касание с каким-либо подмногообразием семейства $\{G\}$, то оно называется огибающим полного порядка этого семейства.

Это определение огибающего подмногообразия $s$-го порядка не является единственно возможным. Другие подходы в теории огибающих освешены в [22]-[24].

В дальнейшем для краткости подмногообразия с параллельной ф.ф.. $\alpha_{s}(s \geqslant 2)$ будем называть $s$-параллельнымми, а с полупараллельной ф.ф. $\alpha_{s}-s$-nолупараллельными (при $s=2$ подразумеваются параллельные и полупараллельные подмногообразия соответственно).

Следующие две теоремы распространяют результат Ю.Г. Лумисте на $s$-параллельные и $s$-полупараллельные $(s \geqslant 3)$ подмногообразия.

Tеорема 1 [14]. $B M_{n}(c)$ т-мерное подмногообразие $M$ класса $C^{\infty}$ является огибающим s-го порядка семейства m-мерных s-параллельных подмногообразий тогда и только тогда, когда оно имеет полупараллельные ф. ф. $\alpha_{s-1} u \alpha_{s}$ при $s \geqslant 3$ и полупараллельную $ф . ф . \alpha_{2}$ при $s=2$. 
Tеорема 2 [14]. Пусть в $M_{n}(c)$ m-мерное подмногообразие $M$ класса $C^{\infty}$ имеет симметрические ф.ф. $\alpha_{3}, \ldots, \alpha_{s-1} \quad(s \geqslant 4)$. Тогда его ф.ф. $\alpha_{s}$ будет симметрической в том и только том случае, когда $M$ является огибающим $(s-2)$-го порядка семейства т-мерных $(s-2)$-параллельных подмногообразий, у каждого из которых все ф. ф. до $(s-2)$-го порядка включительно такжсе являются симметрическими.

В силу импликации [14]

$$
\left(R(X, Y) \cdot \alpha_{s-1}=0, R(X, Y) \cdot \alpha_{s}=0\right) \Rightarrow R(X, Y) \cdot R=0 \quad(s \geqslant 4)
$$

$(s-1)$ - и $s$-полупараллельные подмногообразия составляют новьй класс подмногообразий с внутренней геометрией полусимметрического риманова пространства и в этой связи представляют большой интерес.

Пусть $F(M)$ обозначает класс тензорных полей на подмногообразии $M$ в $M_{n}(c)$, определяемых только второй ф.ф. $\alpha_{2}$ и ее ковариантньми производными произвольного порядка с использованием любых тензорных операций. Классу $F(M)$ принадлежат, в частности, тензор кривизны $R$ римановой связности на $M$, тензор кривизны нормальной связности $R^{\perp}$, тензор Риччи Ric, вектор средней кривизны $H$ и их ковариантные производные любого порядка. Если $M$ и $\widetilde{M}-$ два $m$-мерных подмногообразия в $M_{n}(c)$, то через $T \in F(M)$ и $\widetilde{T} \in F(\widetilde{M})$ будем обозначать соответствующие тензорные поля, т.е. такие тензорные поля, которые выражаются соответственно через вторые ф.ф. $\alpha_{2}$ и $\widetilde{\alpha}_{2}$ (знак $\sim$ обозначает, что объект относится к $\widetilde{M}$ ) и их ковариантные производные различных порядков с использованием одних и тех же тензорных операций. В дальнейшем для произвольных касательных к подмногообразию $M$ векторных полей $X_{1}, \ldots, X_{s}$ ковариантные производные $\bar{\nabla}_{X_{1}} \cdots \bar{\nabla}_{X_{s}} T$ более коротко будем обозначать через $\bar{\nabla}^{s} T$ (при $s=0$ полагаем $\left.\bar{\nabla}^{0} T=T\right)$.

ОПРЕДЕЛЕНИЕ 3 . Пусть в пространстве постоянной кривизны $M_{n}(c) m$-мерные подмногообразия $M$ и $\widetilde{M}$ имеют обшую точку $x$, и пусть $T \in F(M)$ и $\widetilde{T} \in F(\widetilde{M})$ - некоторые соответствующие тензорные поля. Точку $x$ будем назьвать точкой $T$-касания $s$-го $(s \geqslant 0)$ порядка для $M$ и $\widetilde{M}$, если в $x$ совпадают

1) касательные пространства $T_{x}(M)$ и $\widetilde{T}_{x}(\widetilde{M})$,

2) ковариантные производные $\bar{\nabla}^{r} T$ и $\bar{\nabla}^{r} \widetilde{T}$, где $r=0,1, \ldots, s$.

(При $s=0$ будем говорить просто о точке $T$-касания.)

Из определения 1 следует, что каждая точка полного касания автоматически является точкой $T$-касания $s$-го порядка для любого $s \geqslant 0$.

ОПРЕДЕЛЕНИЕ 4. В $M_{n}(c) \quad m$-мерное подмногообразие $M$ будем называть $T$-огибающим $(T \in F(M)) \quad s$-го $(s \geqslant 0)$ порядка для некоторого семейства $m$-мерных подмногообразий $\{G\}$, если в каждой своей точке оно имеет $T$-касание $s$-го порядка с каким-либо подмногообразием этого семейства, причем касание вдоль $M$ переходит от одного подмногообразия семейства к другому. (При $s=0$ будем говорить просто о $T$-огибающем подмногообразии.) 
Если в определениях 3 и 4 тензорное поле $T$ заменить на тензор кривизны $R$ (или на $R^{\perp}$, Ric или $H$ ), то получим определение точки $R$-касания $s$-го порядка, $R$-огибающего подмногообразия $s$-го порядка и т. д.

Если на подмногообразии $M$ в $M_{n}(c)$ ковариантная производная $\bar{\nabla}^{s} T(T \in$ $F(M))$ является параллельной, т.е. $\bar{\nabla}\left(\bar{\nabla}^{s} T\right)=0$, то $M$ будем называть $\bar{\nabla}^{s} T-n a-$ раллельнымм подмногообразием. Если же $\bar{\nabla}^{s} T$ является полупараллельной, т.е. $\bar{R}(X, Y) \cdot \bar{\nabla}^{s} T=0$ для любых касательных к $M$ векторных полей $X, Y$, то $M$ будем называть $\bar{\nabla}^{s} T$-полупараллельным подмногообразием. При $s=0$ получаются определения $T$-параллельного и $T$-полупараллельного подмногообразий.

Следующие теоремы описывают более слабые законы огибания, чем теорема Лумисте и теоремы $1,2$.

ТЕОРема 3 [18]. Пусть в пространстве постоянной кривизны $M_{n}(c)$ m-мерное подмногообразие $M$ является $R$-, $R^{\perp}$ - и $T$-огибающим $(T \in F(M) u$ определяется второй ф.ф. $\alpha_{2}$ с использованием только тензорных операций алгебраического характера) семейства $\{\widetilde{M}\} \quad m$-мерных $\widetilde{T}$-параллельных подмногообразий. Тогда $M$ является T-полупараллельныц подмногообразием.

ЗАмечание 1 . Если в тождестве Риччи для $T$ не участвует какой-либо из тензоров $R$ или $R^{\perp}$, то в теореме 3 соответствующее выражение " $R$-" или " $R^{\perp-}$ " необходимо опустить.

Tеорема 4 [18]. В пространстве постоянной кривизны $M_{n}(c)$ т-мерное T-полупараллельное подмногообразие $M$ ( $T$ такое же, как и в теореме 3$)$ класса $C^{\infty}$ является огибающим полного порядка для некоторого семейства $\{\widetilde{M}\}$ т-мерных $\widetilde{T}$-параллельных подмногообразий.

С учетом замечания 1 теоремы 3 и 4 легко перефразировать для тензоров $R, R^{\perp}$, Ric и вектора $H$.

\section{§2. Формулировка результатов}

В настоящей работе, заключающей цикл исследований автора [14]-[20] по этой проблематике, изложенные в 11 результаты обобщаются и распространяются на ковариантные производные $\bar{\nabla}^{s} T$ произвольного тензорного поля $T \in F(M)$ на подмногообразии $M$ в $M_{n}(c)$.

ТЕОрема 5. Пусть в пространстве постоянной кривизны $M_{n}(c)$ т-мерное подмногообразие $M$ класса $C^{\infty}$ является $R$ - $и R^{\perp}$-огибающим порядка $r=0$ и $T$-огибающим $(T \in F(M))$ порядка $s \geqslant 0$ для некоторого семейства $\{\widetilde{M}\}$ т-мерных $\bar{\nabla}^{s}$ T-параллельных подмногообразий. Тогда $M$ является $\bar{\nabla}^{s-1} T-u$ $\bar{\nabla}^{s}$ T-полупараллельныц при $s \geqslant 1$ и Т-полупараллельныц мри $s=0$.

При формулировке этой теоремы для конкретных тензорных полей необходимо учитывать замечание 1 .

ТЕОРема 6. Пусть в пространстве постоянной кривизны $M_{n}(c)$ m-мерное подмногообразие $M$ класса $C^{\infty}$ является $\bar{\nabla}^{s-1} T-u \bar{\nabla}^{s}$ T-полупараллельныцм $(s \geqslant 0$, при $s=0$ предполагается только $T$-полупараллельность), где $T \in$ 
$F(M)$. Тогда оно является огибающим полного порядка для некоторого семейства $\{\widetilde{M}\} \quad$-мерных $\bar{\nabla}^{s} \widetilde{T}$-параллельных подмногообразий.

Если в теореме $6 T$ совпадает с ф.ф. $\alpha_{s}(s \geqslant 3)$, то можно руководствоваться теоремой 1 , описьваюшей этот случай.

ТЕОРема 7. Пусть в $M_{n}(c)$ на ковариантные производные $\bar{\nabla}_{X_{1}} \ldots \bar{\nabla}_{X_{r}} T(r=2, \ldots, s+1, s \geqslant 2)$ тензорного поля $T \in F(M)$ для любих касательных $\kappa M$ векторных полей $X_{1}, \ldots, X_{r}$ симметричны по әтим аргументам. Для того чтобъ ковариантная производная $\bar{\nabla}_{X_{1}} \cdots \bar{\nabla}_{X_{s+2}} T$ также была симметрична по произвольным аргументам $X_{1}, \ldots, X_{s+2}$, необходимо и достаточно, чтобъ $M$ бъло $R-, \quad R^{\perp}-, \quad \bar{\nabla}^{s} T$-огибающим для некоторого семейства $\{\widetilde{M}\} \quad$-мерных $\bar{\nabla}^{s} \widetilde{T}$-параллельных подмногообразий, у кажсдого из которых ковариантные производные $\bar{\nabla}_{\widetilde{X}_{1}} \cdots \bar{\nabla}_{\widetilde{X}_{r^{\prime}}} \widetilde{T}, r^{\prime}=2, \ldots, s$, симметричны по произвольньм аргументам $\tilde{X}_{1}, \ldots, \widetilde{X}_{r^{\prime}}$.

Теоремы 5-7 окончательно устанавливают законы взаимосвязей, которым подчиняются подмногообразия, несущие параллельные и полупараллельные структуры, выражаемые через вторую ф.ф. и ее ковариантные производные. Теорема Лумисте и теоремы 1-4 описывают частные проявления этих законов.

Классификация и геометрическое описание некоторых классов подмногообразий с параллельными и полупараллельными тензорньпи полями дана в [1], [2], [4], $[5],[7],[8],[10]-[13],[25]$.

\section{§3. Основные формулы и редукция условий}

В настоящем параграфе коротко (подробности см. в [14], [16], [18]) с использованием формализма расслоения адаптированных ортонормреперов подмногообразия приведем основные формулы и уравнения и получим редукцию некоторых условий, необходимых для доказательства теорем $5-7$.

Пусть $\omega=\omega^{a} \otimes e_{a}$ - каноническая 1-форма на $M_{n}(c)$, и пусть $\omega_{b}^{a}$ являются 1-формами связности Леви-Чивита в главном расслоении $O\left(M_{n}(c)\right)$ ортонормреперов $\left(x, e_{1}, \ldots, e_{n}\right)$ в $M_{n}(c)$, где $a, b=1, \ldots, n$. Тогда $\omega_{a}^{b}+\omega_{b}^{a}=0$ и вьполняются следующие структурные уравнения:

$$
d \omega^{a}=\omega^{b} \wedge \omega_{b}^{a}, \quad d \omega_{a}^{b}=\omega_{a}^{d} \wedge \omega_{d}^{b}-c \omega^{a} \wedge \omega^{b}
$$

Пусть $M$ является $m$-мерным подмногообразием класса $C^{\infty}$ в $M_{n}(c)$. Тогда расслоение $O\left(M_{n}(c)\right)$ может быть приведено к главному расслоению $O\left(M, M_{n}(c)\right)$ адаптированных ортонормреперов $\left(x, e_{1}, \ldots, e_{m}, e_{m+1}, \ldots, e_{n}\right)$, где векторы $e_{i}$ $(i, j, \ldots=1, \ldots, m)$ принадлежат касательному пространству $T_{x}(M)$, а векторы $e_{\alpha}(\alpha, \beta, \ldots=m+1, \ldots, n)$ - нормальному пространству $T_{x}^{\perp}(M), x \in M$ (подробности см. в [26; гл. VII, §1]). В силу этого по известной схеме (см. [7], 
[14], [18]) получим

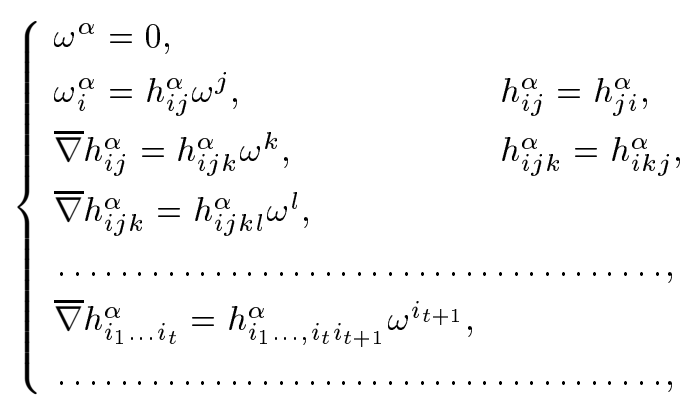

где $\bar{\nabla}$ обозначает связность Ван дер Вардена-Бортолотти, а функции $h_{i_{1} \ldots i_{t}}^{\alpha}$ $(t \geqslant 2)$, определяемые уравнениями системы (3.1), являются компонентами ф.ф. $\alpha_{t}$. Все уравнения в (3.1), начиная с третьего, в развернутой записи имеют следуюший вид:

$$
d h_{i_{1} \ldots i_{t}}^{\alpha}+h_{i_{1} \ldots i_{t}}^{\beta} \omega_{\beta}^{\alpha}-h_{k i_{2} \ldots i_{t}}^{\alpha} \omega_{i_{1}}^{k}-\ldots-h_{i_{1} \ldots k}^{\alpha} \omega_{i_{t}}^{k}=h_{i_{1} \ldots i_{t} i_{t+1}}^{\alpha} \omega^{i_{t+1}} .
$$

Формы $\alpha_{t}$ являются $T^{\perp}(M)$-значными (через $T^{\perp}(M)$ обозначаем нормальное расслоение) и действуют по правилу

$$
\alpha_{t}:\left(X_{1}, \ldots, X_{t}\right) \rightarrow h_{i_{1} \ldots i_{t}}^{\alpha} X_{1}^{i_{1}} \cdots X_{t}^{i_{t}} e_{\alpha}
$$

где $X_{r}=X_{r}^{i} e_{i}, r=1, \ldots, t$. Из (3.1) следует, что $\alpha_{t}=\bar{\nabla} \alpha_{t-1}(t \geqslant 3)$.

Дифференцируя внешним образом уравнение (3.2), получаем условие его интегрируемости:

$$
h_{i_{1} \ldots i_{t} k l}^{\alpha} \omega^{k} \wedge \omega^{l}=h_{k \ldots i_{t}}^{\alpha} \Omega_{i_{1}}^{k}+\cdots+h_{i_{1} \ldots k}^{\alpha} \Omega_{i_{t}}^{k}-h_{i_{1} \ldots i_{t}}^{\beta} \Omega_{\beta}^{\alpha} .
$$

Здесь

$$
\begin{aligned}
& \Omega_{i}^{j}=d \omega_{i}^{j}-\omega_{i}^{k} \wedge \omega_{k}^{j}=-\left(\sum_{\alpha} h_{i[k}^{\alpha} h_{l] j}^{\alpha}+c \delta_{i[k} \delta_{l] j}\right) \omega^{k} \wedge \omega^{l} \\
& \Omega_{\alpha}^{\beta}=d \omega_{\alpha}^{\beta}-\omega_{\alpha}^{\gamma} \wedge \omega_{\gamma}^{\beta}=-\sum_{i} h_{i[k}^{\alpha} h_{l] i}^{\beta} \omega^{k} \wedge \omega^{l}
\end{aligned}
$$

являются 2-формами связности $\bar{\nabla}=\nabla \oplus \nabla^{\perp}$, где $\nabla$ обозначает риманову связность на подмногобразии $M$, определенную 1 -формами $\omega_{i}^{j}$, а $\nabla^{\perp}-$ нормальную связность, определенную 1-форомами $\omega_{\beta}^{\alpha}$.

В (3.4) и (3.5) коэффициенты

$$
R_{i k l}^{j}=-\left(\sum_{\alpha} h_{i[k}^{\alpha} h_{l] j}^{\alpha}+c \delta_{i[k} \delta_{l] j}\right), \quad R_{\alpha k l}^{\beta}=-\sum_{i} h_{i[k}^{\alpha} h_{l] i}^{\beta}
$$

при $\omega^{k} \wedge \omega^{l}$ являются компонентами тензоров кривизны $R$ и $R^{\perp}$ связностей $\nabla$ и $\nabla^{\perp}$ соответственно. При $R=0$ говорят о локально евклидовом подмногообразии, а при $R^{\perp}=0$ - о подмногообразии с плоской нормальной связностью. 
Учитывая (3.3), условия интегрируемости системы (3.1) можем записать в следующем виде:

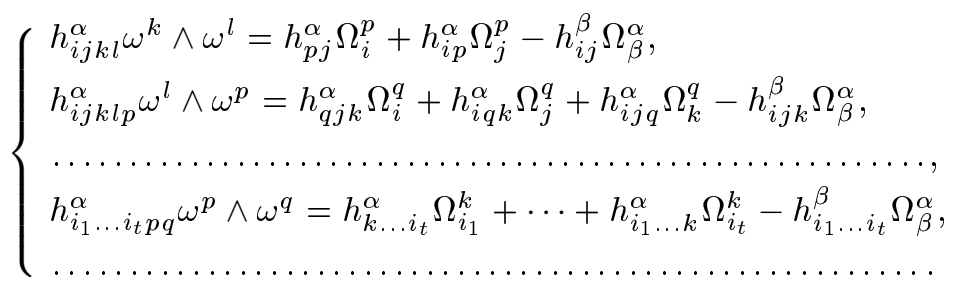

ЗАмЕчАниЕ 2. Отметим, что системы (3.1) и (3.7) всегда конечны, так как в силу конечности $n$ в каждой точке $x \in M$ пространство $T_{x}\left(M_{n}(c)\right)$ при увеличении $t$ необходимо заполняется или заполнение останавливается на некотором подпространстве (см. определение нормальных пространств).

Пусть $T \in F(M)$ - некоторое тензорное поле на подмногообразии $M$, компоненты $T_{i j}^{\alpha}$ которого (такое строение компонент не ограничивает общности рассуждений, ибо увеличение числа нижних и верхних индексов приведет только к усложнению выкладок) выражаются через $h_{i_{1} \ldots i_{t}}^{\alpha}(t \geqslant 2)$ с использованием произвольных тензорных операций. Через $T_{i j, i_{1} \ldots i_{r}}^{\alpha}$ будем обозначать компоненты ковариантных производных $\bar{\nabla}^{r} T$. Они определяются из системы

$$
\left\{\begin{array}{l}
\bar{\nabla} T_{i j}^{\alpha}=T_{i j, i_{1}}^{\alpha} \omega^{i_{1}} \\
\bar{\nabla} T_{i j, i_{1}}^{\alpha}=T_{i j, i_{1} i_{2}}^{\alpha} \omega^{i_{2}} \\
\ldots \ldots \ldots \ldots \ldots \ldots \ldots \ldots \ldots \ldots \ldots \ldots \ldots \ldots \ldots \ldots \ldots
\end{array}\right.
$$

где левые части раскрываются по схеме (3.2).

Дифференцируя внешним образом уравнение

$$
d T_{i j, i_{1} \ldots i_{s}}^{\alpha}+T_{i j, i_{1} \ldots i_{s}}^{\beta} \omega_{\beta}^{\alpha}-T_{k j, i_{1} \ldots i_{s}}^{\alpha} \omega_{i}^{k}-\ldots-T_{i j, i_{1} \ldots k}^{\alpha} \omega_{i_{s}}^{k}=T_{i j, i_{1} \ldots i_{s} k}^{\alpha} \omega^{k}
$$

получаем условие его интегрируемости в следующем виде:

$$
T_{i j, i_{1} \ldots i_{s} k l}^{\alpha} \omega^{k} \wedge \omega^{l}=T_{k j, i_{1} \ldots i_{s}}^{\alpha} \Omega_{i}^{k}+T_{i k, i_{1} \ldots i_{s}}^{\alpha} \Omega_{j}^{k}+\cdots+T_{i j, i_{1} \ldots k}^{\alpha} \Omega_{i_{s}}^{k}-T_{i j, i_{1} \ldots i_{s}}^{\beta} \Omega_{\beta}^{\alpha}
$$

Следовательно, система

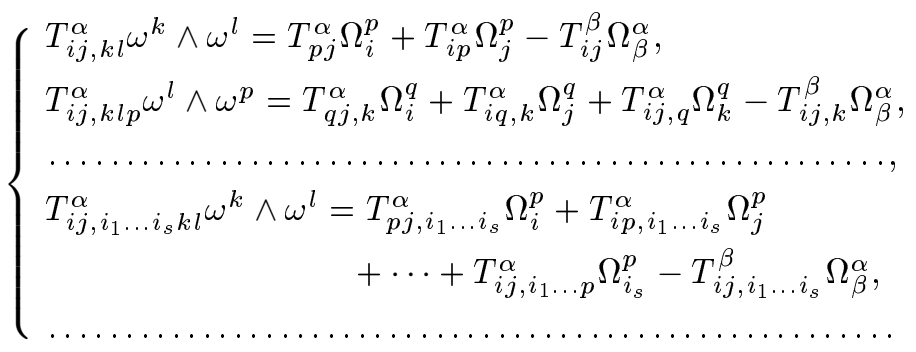


дает условия интегрируемости системы (3.8).

Условия параллельности и полупараллельности ковариантной производной $\bar{\nabla}^{s} T$ аналитически выражаются соответственно следующими уравнениями:

$$
\begin{gathered}
d T_{i j, i_{1} \ldots i_{s}}^{\alpha}+T_{i j, i_{1} \ldots i_{s}}^{\beta} \omega_{\beta}^{\alpha}-T_{k j, i_{1} \ldots i_{s}}^{\alpha} \omega_{i}^{k}-T_{j k, i_{1} \ldots i_{s}}^{\alpha} \omega_{j}^{k} \\
-T_{i j, k \ldots i_{s}}^{\alpha} \omega_{i_{1}}^{k}-\ldots-T_{i j, i_{1} \ldots k}^{\alpha} \omega_{i_{s}}^{k}=0 \\
\left.T_{k j, i_{1} \ldots i_{s}}^{\alpha} \Omega_{i}^{k}+T_{i k, i_{1} \ldots i_{s}}^{\alpha} \Omega_{j}^{k}+T_{i j, k \ldots i_{s}}^{\alpha} \Omega_{i_{1}}^{k}+\cdots+T_{i j, i_{1} \ldots k}^{\alpha} \Omega_{i_{s}}^{k}-T_{i j, i_{1} \ldots i_{s}}^{\beta} \Omega_{\beta}^{\alpha}=0.11\right)
\end{gathered}
$$

Условие (3.11) коротко можно записать в виде $\bar{\nabla} T_{i j, i_{1} \ldots i_{s}}^{\alpha}=0$, что равносильно $T_{i j, i_{1} \ldots i_{s} i_{s+1}}^{\alpha}=0$. Из (3.9) следует, что (3.12) равносильно симметричности компонент $T_{i j, i_{1} \ldots i_{s} k l}^{\alpha}$ по двум последним нижним индексам.

Пусть $\bar{\nabla} \bar{\nabla}^{s} T=0$. Тогда $T_{i j, i_{1} \ldots i_{s} k}^{\alpha}=0$ и, следовательно, $T_{i j, i_{1} \ldots i_{s} k l}^{\alpha}=0$. Из первого равенства следует полупараллельность $\bar{\nabla}^{s-1} T$, а из второго - полупараллельность $\bar{\nabla}^{s} T$.

Таким образом, если ковариантная производная $\bar{\nabla}^{s} T$ является параллельной, то как $\bar{\nabla}^{s-1} T$, так и $\bar{\nabla}^{s} T$ являются полупараллельными и выполняются следующие условия:

$$
\left\{\begin{array}{c}
T_{k j, i_{1} \ldots i_{s-1}}^{\alpha} \Omega_{i}^{k}+T_{i k, i_{1} \ldots i_{s-1}}^{\alpha} \Omega_{j}^{k}+T_{i j, k \ldots i_{s-1}}^{\alpha} \Omega_{i_{1}}^{k} \\
+\cdots+T_{i j, i_{1} \ldots k}^{\alpha} \Omega_{i_{s-1}}^{k}-T_{i j, i_{1} \ldots i_{s-1}}^{\beta} \Omega_{\beta}^{\alpha}=0 \\
T_{k j, i_{1} \ldots i_{s}}^{\alpha} \Omega_{i}^{k}+T_{i k, i_{1} \ldots i_{s}}^{\alpha} \Omega_{j}^{k}+T_{i j, k \ldots i_{s}}^{\alpha} \Omega_{i_{1}}^{k} \\
+\cdots+T_{i j, i_{1} \ldots k}^{\alpha} \Omega_{i_{s}}^{k}-T_{i j, i_{1} \ldots i_{s}}^{\beta} \Omega_{\beta}^{\alpha}=0
\end{array}\right.
$$

Из (3.9) следует, что функции $T_{i j, k l}^{\alpha}, T_{i j, k l p}^{\alpha}, \ldots, T_{i j, i_{1} \ldots i_{s+2}}^{\alpha}$ одновременно будут симметрическими по всем нижним индексам, стоящим после запятой (что равносильно одновременной полупараллельности тензоров $\left.T_{i j}^{\alpha}, T_{i j, k}^{\alpha}, \ldots, T_{i j, i_{1} \ldots i_{s}}^{\alpha}\right)$, тогда и только тогда, когда выполняется следуюшая система условий:

$$
\left\{\begin{array}{l}
T_{p j}^{\alpha} \Omega_{i}^{p}+T_{i p}^{\alpha} \Omega_{j}^{p}-T_{i j}^{\beta} \Omega_{\beta}^{\alpha}=0 \\
T_{p j, k}^{\alpha} \Omega_{i}^{p}+T_{i p, k}^{\alpha} \Omega_{j}^{p}+T_{i j, p}^{\alpha} \Omega_{k}^{p}-T_{i j, k}^{\beta} \Omega_{\beta}^{\alpha}=0 \\
\ldots \ldots \ldots \ldots \ldots \ldots \ldots \ldots \ldots \ldots \ldots \ldots \ldots \ldots \ldots \ldots \ldots \ldots \ldots \ldots \ldots \ldots \ldots \ldots \ldots \ldots \ldots \\
T_{p j, i_{1} \ldots i_{s}}^{\alpha} \Omega_{i}^{p}+T_{i p, i_{1} \ldots i_{s}}^{\alpha} \Omega_{j}^{p}+T_{i j, p \ldots i_{s}}^{\alpha} \Omega_{i_{1}}^{p} \\
\quad+\cdots+T_{i j, i_{1} \ldots p}^{\alpha} \Omega_{i_{s}}^{p}-T_{i j, i_{1} \ldots i_{s}}^{\beta} \Omega_{\beta}^{\alpha}=0 .
\end{array}\right.
$$

Очевидно, что если подмногообразие является локально евклидовым и имеет плоскую нормальную связность, то все ковариантные производные $T_{i j, i_{1} \ldots i_{s}}^{\alpha}(s \geqslant 2)$ тензорного поля $T_{i j}^{\alpha}$ автоматически будут симметрическими по всем нижним индексам, стоящим после запятой, и, следовательно, будут полупараллельными. 


\section{§4. Доказательство теоремы 5}

Пусть выполняются условия теоремы 5 , и пусть $\widetilde{M}$ - подмногообразие семейства $\{\widetilde{M}\}$, с которьм $M$ имеет касание в точке $x$. Тогда в этой точке в общем для $M$ и $\widetilde{M}$ адаптированном ортонормрепере совпадают

1) $R_{i k l}^{j}$ и $\widetilde{R}_{i k l}^{j}$,

2) $R_{\alpha k l}^{\beta}$ и $\widetilde{R}_{\alpha k l}^{\beta}$,

3) $T_{i j, i_{1} \ldots i_{t}}^{\alpha}$ и $\widetilde{T}_{i j, i_{1} \ldots i_{t}}^{\alpha}, t=0,1, \ldots, s$,

где $\widetilde{T}$ - тензорное поле на $\widetilde{M}$, соответствующее $T$. Так как подмногообразие $\widetilde{M}$ является $\bar{\nabla}^{s} \widetilde{T}$-параллельньм, то тензорные поля $\bar{\nabla}^{s-1} \widetilde{T}$ и $\bar{\nabla}^{s} \widetilde{T}$ являются полупараллельными и их компоненты $\widetilde{T}_{i j, i_{1} \ldots i_{s-1}}^{\alpha}$ и $\widetilde{T}_{i j, i_{1} \ldots i_{s}}^{\alpha}$ удовлетворяют условиям (3.13), записанным для $\widetilde{M}$, которые равносильны (см. (3.4)-(3.6)) системе

$$
\left\{\begin{array}{c}
\widetilde{T}_{k j, i_{1} \ldots i_{s-1}}^{\alpha} \widetilde{R}_{i p q}^{k}+\widetilde{T}_{i k, i_{1} \ldots i_{s-1}}^{\alpha} \widetilde{R}_{j p q}^{k} \\
\quad+\cdots+\widetilde{T}_{i j, i_{1} \ldots k}^{\alpha} \widetilde{R}_{i_{s-1} p q}^{k}-\widetilde{T}_{i j, i_{1} \ldots i_{s-1}}^{\beta} \widetilde{R}_{\beta p q}^{\alpha}=0 \\
\widetilde{T}_{k j, i_{1} \ldots i_{s}}^{\alpha} \widetilde{R}_{i p q}^{k}+\widetilde{T}_{i k, i_{1} \ldots i_{s}}^{\alpha} \widetilde{R}_{j p q}^{k} \\
+\cdots+\widetilde{T}_{i j, i_{1} \ldots k}^{\alpha} \widetilde{R}_{i_{s} p q}^{k}-\widetilde{T}_{i j, i_{1} \ldots i_{s}}^{\beta} \widetilde{R}_{\beta p q}^{\alpha}=0
\end{array}\right.
$$

В силу алгебраического характера этих условий и совпадения в точке $x$ указанных выше тензоров $T_{i j, i_{1} \ldots i_{s-1}}^{\alpha}$ и $T_{i j, i_{1} \ldots i_{s}}^{\alpha}$ также удовлетворяют в точке $x$ аналогичным условиям. Так как точка $x \in M$ произвольная, а условия (4.1) имеют тензорный характер, то мы заключаем, что ковариантные производные $\bar{\nabla}^{s-1} T$ и $\bar{\nabla}^{s} T$ являются полупараллельньми.

\section{§5. Доказательство теоремы 6}

Доказательство будем проводить методом подвижного репера Картана с использованием теории вполне интегрируемых дифференциальных систем Фробениуса-Картана. Идея доказательства такая же, как и в [14], [16], [18], однако имеются и сушественные различия.

Пусть $m$-мерное подмногообразие $M$, на котором задано тензорное поле $T$ с полупараллельными ковариантными производньми $\bar{\nabla}^{s-1} T$ и $\bar{\nabla}^{s} T$, в расслоении адаптированных ортонормреперов в $M_{n}(c)$ определяются вполне интегрируемой системой (3.1). Последнее означает, что выполняются также условия (3.7). Пусть $x \in M$, и пусть $E_{n}$ обозначает касательное евклидово пространство к $M_{n}(c)$ в точке $x$, а $T_{x}(M)$ - касательное пространство к $M$ в $x$. Обозначим через $L$ многообразие всех наборов $\left(x, T_{x}(M), \alpha_{2}, \alpha_{3}, \ldots, \alpha_{t}, \ldots\right)$ в $E_{n}$, где $\alpha_{t}-t$-я $(t=2, \ldots, s, \ldots)$ ф.ф. подмногообразия $M$, которая отображает произведение $\underbrace{T_{x}(M) \times \cdots \times T_{x}(M)}_{t}$ в $T_{x}^{\perp}(M)$ (ортогональное дополнение к $T_{x}(M)$ в $\left.E_{n}\right)$. В силу замечания 2 число ф.ф. в наборах конечно и одно и то же для всех наборов. Это число будем называть длиной набора. Если дополнить такой набор адаптированньм ортонормированным репером $\left(x, e_{1}, \ldots, e_{m}, e_{m+1}, \ldots, e_{n}\right)$, где $e_{i} \in T_{x}(M), e_{\alpha} \in T_{x}^{\perp}(M)$ $(i, j, \ldots=1, \ldots, m ; \alpha, \beta, \ldots=m+1, \ldots, n)$, то получим реперированный набор. 
Многообразие всех реперированных наборов указанной конечной длины в $E_{n}$ обозначим через $L^{\mathrm{fr}}$. Локальными координатами в $L^{\mathrm{fr}}$ являются: $x^{a}-$ координаты $(a=1, \ldots, n)$ точки $x$, параметры $\varphi^{u}(u=1, \ldots, n(n-1) / 2)$ таких ортогональных матриц $A$ в $O(n, R)$, которые преобразуют базис $\left\{\partial / \partial x^{a}\right\}$ в базис $\left\{e_{a}\right\}$ после стандартной ортогонализации, компоненты $h_{i_{1} \ldots i_{t}}^{\alpha}$ форм $\alpha_{t}(t \geqslant 2)$ в последнем базисе.

На многообразии $L^{\text {fr }}$ рассмотрим дифференциальную систему

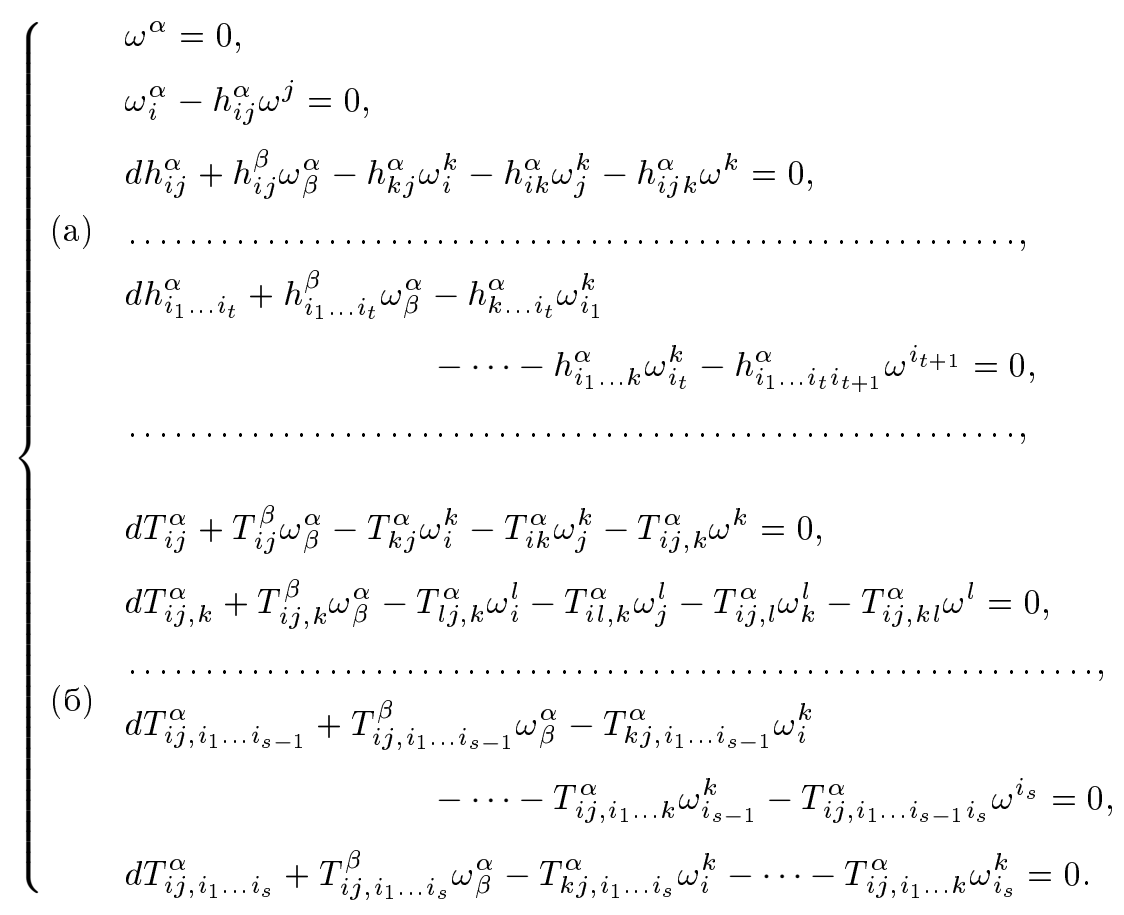

Эта система состоит из подсистем (а) и (б). Подсистема (а) совпадает с системой (3.1), для которой выполняются условия интегрируемости (3.7). В подсистеме (б) $T_{i j}^{\alpha}$ являются компонентами тензорного поля $T$, а $T_{i j, i_{1} \ldots i_{t}}^{\alpha}-$ компонентами ковариантной производной $\bar{\nabla}^{t} T$. По условию теоремы они выражаются через $h_{i_{1} \ldots i_{r}}^{\alpha}(r \geqslant 2)$ с помощью тензорных операций. Будем предполагать, что функции $T_{i j, i_{1} \ldots i_{r}}^{\alpha}, r=0,1, \ldots, s$, удовлетворяют

1) только второму уравнению системы (3.13) при $s=0$ (первое уравнение теряет смысл),

2) системе (3.13) при $s=1$,

3) системе (3.13) и первым $s-1$ уравнениям системы (3.10) при $s>1$.

Предположим также, что $R_{i k l}^{j}$ и $R_{\alpha k l}^{\beta}$ определяются через $h_{i j}^{\alpha}$ по формулам (3.6), а через них и $\Omega_{i}^{j}, \Omega_{\alpha}^{\beta}$ по формулам (3.4) и (3.5) соответственно. Тогда путем прямого вычисления получим, что система (5.1) вполне интегрируема.

Два реперированных набора будем называть әквивалентными , если $e_{j}^{\prime}=A_{i}^{j} e_{j}$, $e_{\alpha}^{\prime}=A_{\alpha}^{\beta} e_{\beta}$, где $\left\|A_{i}^{j}\right\| \in O(m, R),\left\|A_{\alpha}^{\beta}\right\| \in O(n-m, R)$. Тогда для любого $t \geqslant 2$ будем иметь

$$
h_{i_{1} \ldots i_{t}}^{\prime \alpha}=h_{j_{1} \ldots j_{t}}^{\beta} A_{\beta}^{\alpha} A_{i_{1}}^{j_{1}} \cdots A_{i_{t}}^{j_{t}} .
$$


Так как $T_{i j}^{\alpha}$ и $T_{i j, i_{1} \ldots i_{r}}^{\alpha}$ выражаются через $h_{i_{1} \ldots i_{t}}^{\alpha}$ с помощью тензорных операций, то они будут преобразовываться точно по такому же закону, что и (5.2). Поэтому введенная выше эквивалентность определяет отображение $L^{\mathrm{fr}} \rightarrow L$, которое проектирует систему (5.1) в корректно определенную вполне интегрируемую дифференциальную систему на $L$. Отсюда следует, что для каждого фиксированного набора из $L$ локально сушествует интегральное подмногообразие этой дифференциальной системы, которое имеет максимально возможную размерность, равную размерности инволюнтного распределения на $L$, соответствуюшего этой системе, т.е. $m$. Уравнения подсистемы (а) показывают, что фундаментальными формами этого подмногообразия являются $\alpha_{2}, \alpha_{3}, \ldots$ и т.д. Последнее уравнение подсистемы (б) показывает, что на этом подмногообразии тензорное поле $T_{i j, i_{1} \ldots i_{s}}^{\alpha}$ является параллельным.

Пусть фиксированный набор $\left(x, T_{x}(M), \alpha_{2 x}, \ldots, \alpha_{t x}, \ldots\right)$ подмногообразия $M$ определяет, как это было описано выше, некоторое $m$-мерное подмногообразие $\widetilde{M}$, порожденное системой (5.1) и проходящее через $x$. Так как в точке $x \in M \cap \widetilde{M}$ наборы $\left(x, T_{x}(M), \alpha_{2 x}, \ldots, \alpha_{t x}, \ldots\right)$ и $\left(x, T_{x}(\widetilde{M}), \widetilde{\alpha}_{2 x}, \ldots, \widetilde{\alpha}_{t x}, \ldots\right)$ совпадают по построению, то $M$ является огибающим полного порядка для всех таких $\widetilde{M}$.

\section{§6. Доказательство теоремы 7}

Необходимость. Пусть на подмногообразии $M$ ковариантные производные $T_{i j, i_{1} \ldots i_{r}}^{\alpha}(r=2, \ldots, s+1$, где $s \geqslant 2)$ симметричны по индексам $i_{1}, \ldots, i_{r}$, что равносильно выполнению условий (3.14). Рассуждая так же, как и при доказательстве теоремы 6, получаем, что дифференциальная система (5.1) при вьполнении условий (3.7) и (3.14) вполне интегрируема. Следовательно, она определяет, как это было установлено при доказательстве теоремы 6 , семейство $m$-мерных $\bar{\nabla}^{s} \widetilde{T}$-параллельных подмногообразий $\{\widetilde{M}\}$, для которого $M$ является огибающим порядка $p \geqslant 2$, а следовательно, $R$-и $R^{\perp}$-огибающим. Из (5.1) следует, что для подмногообразия $\widetilde{M} \in\{\widetilde{M}\}$ функции $h_{i_{1} \ldots i_{t}}^{\alpha}, t \geqslant 2$, будут компонентами его фундаментальной формы $\widetilde{\alpha}_{t}$. Функции $T_{i j}^{\alpha}$ будут компонентами соответствующего тензорного поля $\widetilde{T}$, а $T_{i j, i_{1} \ldots i_{r^{\prime}}}^{\alpha}-$ компонентами ковариантной производной $\bar{\nabla}^{r^{\prime}} \widetilde{T}$, $r^{\prime}=1, \ldots, s$. Тогда точка касания $M$ и $\widetilde{M}$ будет точкой $\bar{\nabla}^{s} T$-касания, и так как $T_{i j, i_{1} \ldots i_{r^{\prime}}}^{\alpha}$ симметричны по индексам $i_{1}, \ldots, i_{r^{\prime}}$, то необходимость условий теоремы доказана.

Достаточность. Пусть $T_{i j, i_{1} \ldots i_{r}}^{\alpha}(r=2, \ldots, s+1$, где $s \geqslant 2)$ симметричны по индексам $i_{1}, \ldots, i_{r}$, и пусть $M$ является $R-, R^{\perp}$-и $\bar{\nabla}^{s} T$-огибаюшим для некоторого семейства $m$-мерных $\bar{\nabla}^{s} T$-параллельных подмногообразий $\{\widetilde{M}\}$, причем если $\widetilde{M} \in$ $\{\widetilde{M}\}$, то компоненты $\widetilde{T}_{i j, i_{1} \ldots i_{r^{\prime}}}^{\alpha}\left(r^{\prime}=2, \ldots, s\right)$ ковариантных производных соответствующего тензорного поля $\widetilde{T}_{i j}^{\alpha}$ симметричны по индексам $i_{1}, \ldots, i_{r^{\prime}}$. Покажем, что $T_{i j, i_{1} \ldots i_{s+2}}^{\alpha}$ симметричны по индексам $i_{1}, \ldots, i_{s+2}$. Так как $\bar{\nabla} T_{i j, i_{1} \ldots i_{s+1}}^{\alpha}=$ $T_{i j, i_{1} \ldots i_{s+1} i_{s+2}}^{\alpha} \omega^{i_{s+2}}$, то $T_{i j, i_{1} \ldots i_{s+2}}^{\alpha}$ симметричны по индексам $i_{1}, \ldots, i_{s+1}$. Так как тензор $\bar{\nabla}^{s} \widetilde{T}$ является параллельньм, то он автоматически является полупараллельным. Следовательно, его компоненты $\widetilde{T}_{i j, i_{1} \ldots i_{s}}^{\alpha}$ удовлетворяют второму уравнению системы (4.1). В силу алгебраического характера этого уравнения и совпадения в точке касания тензоров $R$ и $\widetilde{R}, R^{\perp}$ и $\widetilde{R}^{\perp}, \bar{\nabla}^{s} T$ и $\bar{\nabla}^{s} \widetilde{T}$ компоненты $T_{i j, i_{1} \ldots i_{s}}^{\alpha}$ 
будут удовлетворять точно такому же уравнению. Это равносильно полупараллельности $T_{i j, i_{1} \ldots i_{s}}^{\alpha}$, что также равносильно симметричности $T_{i j, i_{1} \ldots i_{s+2}}^{\alpha}$ по двум последним нижним индексам.

\section{Список литературы}

1. Deprez J. Semi-parallel hypersurfaces // Rend. Sem. Mat. Univ. Politec. Torino. 1986. V. 44. P. 303-316.

2. Dillen F., Nölker S. Semi-parallelity, multi-rotation surfaces and the helix-property // J. Reine Angew. Math. 1993. V. 435. P. 33-63.

3. Asperti A. C., Mercuri F. Semi-parallel immersions into space form // Boll. Unione Mat. Ital. Sez. B. Artic. Ric. Mat. (8). 1994. V. 7. № 4. P. 833-895.

4. Lumiste $\ddot{U}$. Semi-parallel time-like surfaces in Lorentzian spacetime form // Differential Geom. Appl. 1997. V. 7. P. 59-74.

5. Lumiste $\ddot{U}$. Isometric semiparallel immersions of two-dimensional Riemannian manifolds into pseudo-Euclidean spaces // New developments in differential geometry. Proc. Conf. Budapest, July 27-30, 1996. Dordrecht: Kluwer Acad. Publ., 1999. P. 243-264.

6. Lumiste $\ddot{U}$. Semi-symmetric submanifold as the second order envelope of symmetric submanifolds // Proc. Estonian Acad. Phys. Math. 1990. V. 39. P. 1-8.

7. Лумисте Ю.Г. Полусимметрические подмногообразия // Итоги науки и техн. Проблемы геометрии. Т. 23. М.: ВИНИТИ, 1991. С. 3-28.

8. Мирзоян B. A. Ric-полусимметрические подмногообразия // Итоги науки и техн. Проблемы геометрии. Т. 23. М.: ВИНИТИ, 1991. С. 29-66.

9. Мирзоян $B . A$. Полусимметрические подмногообразия и их разложение в произведение // Изв. вузов. Сер. матем. 1991. №9. С. 29-38.

10. Lumiste $\ddot{U}$. Submanifolds with parallel fundamental form // Handbook of differential geometry / ed. F. Dillen and L. Verstraelen. V. 1. Amsterdam: North-Holland, 2000. P. $779-864$.

11. Ferus D. Symmetric submanifolds of Euclidean space // Math. Ann. 1980. V. 247. P. 81-93.

12. Takeuchi M. Parallel submanifolds of space forms // Manifolds and Lie groups Papers in honor of Y. Matsushima. Basel: Birkhäuser, 1981. P. 429-447. (Progr. Math. V. 14.)

13. Backes E., Reckziegel H. On symmetric submanifolds of spaces of constant curvature // Math. Ann. 1983. V. 263. № 4. P. 419-433.

14. Мирзоян B. A. S-полупараллельные подмногообразия в пространствах постоянной кривизны как огибающие $s$-параллельных подмногообразий // Изв. НАН Армении. Сер. матем. 1996. Т. 31. № 5. С. 44-56.

15. Мирзоян B. A. О $k$-полупараллельных подмногообразиях // Международная геометрическая школа-семинар памяти Н. В. Ефимова. Абрау-Дюрсо, 27 сент.-4 окт. 1996 г. Тезисы докладов. Ростов-на-Дону, 1996. С. 141-142.

16. Мирзоян В. А. Подмногообразия с симметрическими фундаментальнњми формами высшего порядка как огибающие // Изв. вузов. Сер. матем. 1997. № 9. С. 35-40.

17. Мирзоян B. A. Подмногообразия с полупараллельньми фундаментальными формами высшего порядка как огибающие // Изв. вузов. Сер. матем. 1998. № 8. С. 79-80.

18. Мирзоян В. А. Обобщения теоремы Ю. Лумисте о полупараллелњных подмногообразиях // Изв. НАН Армении. Сер. матем. 1998. Т. 33. № 1. С. 53-64.

19. Мирзоян В. А. Полупараллельные структуры как огибающие // Международная геометрическая школа-семинар памяти Н. В. Ефимова. Абрау-Дюрсо, 5-11 сент. 1998 г. Тезисы докладов. Ростов-на-Дону, 1998. С. 56-57.

20. Мирзоян B. A. Римановы риччи-полусимметрические многообразия и их изометрические погружения в пространства постоянной кривизны // Дис. . . докт. физ.-матем. наук. Ереван: ГИУА, 1998. 
21. Алексеевский Д. В., Виноградов А. М., Льчагин В.В. Основные идеи и понятия дифференциальной геометрии // Итоги науки и техн. Соврем. пробл. матем. Фундам. направления. Т. 28. М.: ВИНИТИ, 1988. С. 5-289.

22. Фавар Ж. Курс локальной дифференциальной геометрии. М.: ИЛ, 1960.

23. Залгаллер В. А. Теория огибающих. М.: Наука, 1975.

24. Kowalski O., Nikčević S. Z. Contact homogeneity and envelopes of Riemannian metrics // Beiträge Algebra Geom. 1998. V. 39. № 1. P. 155-167.

25. Мирзоян B. A. Классификация Ric-полупараллельных гиперповерхностей в евклидовых пространствах // Матем. сб. 2000. Т. 191. №9. С. 65-80.

26. Кобаяси Ш., Номидзу К. Основы дифференциальной геометрии. Т. 2. М.: Наука, 1981.

Государственный инженерньй университет Армении,

Поступила в редакцию

г. Ереван, Армения

25.07.2001 